\title{
Non-aqueous phase liquid spreading during soil vapor extraction
}

\author{
Timothy J. Kneafsey $a, b,{ }^{*}$ and James R. Hunt ${ }^{b}$ \\ aEarth Sciences Division, Lawrence Berkeley National Laboratory, University of California, \\ Berkeley, CA 94720, USA \\ bDepartment of Civil and Environmental Engineering, University of California at Berkeley, Berkeley, \\ CA 94720-1710, USA
}

\begin{abstract}
Many non-aqueous phase liquids (NAPLs) are expected to spread at the air - water interface, particularly under non-equilibrium conditions. In the vadose zone, this spreading should increase the surface area for mass transfer and the efficiency of volatile NAPL recovery by soil vapor extraction (SVE). Observations of spreading on water wet surfaces led to a conceptual model of oil spreading vertically above a NAPL pool in the vadose zone. Analysis of this model predicts that spreading can enhance the SVE contaminant recovery compared to conditions where the liquid does not spread. Experiments were conducted with spreading volatile oils hexane and heptane in wet porous media and capillary tubes, where spreading was observed at the scale of centimeters. Within porous medium columns up to a meter in height containing stagnant gas, spreading was less than ten centimeters and did not contribute significantly to hexane volatilization. Water film thinning and oil film pinning may have prevented significant oil film spreading, and thus did not enhance SVE at the scale of a meter. The experiments performed indicate that volatile oil spreading at the field scale is unlikely to contribute significantly to the efficiency of SVE.
\end{abstract}

\section{Keywords}

Unsaturated zone; Contaminant transport; Soil vapor extraction; Film flow

\section{Introduction}

Many non-aqueous phase liquids (NAPLs) are of environmental concern when released near the earth's surface. These liquids are composed of pure or a mixture of compounds, some of which may exhibit toxic and/or carcinogenic properties to humans and other biota and can reach target populations by drinking water or inhalation pathways. Understanding the mechanisms of NAPL migration is important to better understand their subsurface transport and to design efficient cleanup schemes. Within the vadose zone, NAPL migration is controlled by a number of processes including flow by buoyant and capillary forces, liquid spreading at air-water interfaces, and evaporation (Conrad et al., 1992). The efficiency of NAPL recovered by soil vapor extraction (SVE) is dependent on these processes. Current models for SVE are based on air flow through regions containing emplaced NAPL where local equilibrium is often assumed (Armstrong et al., 1994; Baehr et al., 1989; Kearl et al., 1991; Lingineni and Dhir, 1992; Massmann et al., 2000). The efficiency of SVE for NAPL floating on the water table and the effect of NAPL spreading is the focus of this research. 
There is a dramatic difference between the practice of SVE and a fundamental understanding of mechanisms in the subsurface environment. Analysis of field data is limited to quantifying the cumulative recovery of contaminants and extraction rate to demonstrate effectiveness and to argue when sufficient mass has been recovered (Johnson et al., 1990). Many models consider only volatile organic chemical partitioning between air and soil water phases (Fischer et al., 1996) and others have included various adsorption processes, both under equilibrium and nonequilibrium conditions (Barnes and McWhorter, 2000; Brusseau, 1991; Goltz and Oxley, 1994; Poulsen et al., 1998). Some models have included the existence of NAPLs as a fourth component of the subsurface that may or may not be in equilibrium with the soil gas. Massmann et al. (2000) specifically addressed the uncertainties in subsurface geology, emplacement processes, gas transport and mass removal processes. The model assumed a single component NAPL in local equilibrium with the soil gas. Wilkins et al. (1995) included NAPL spreading at the air-water interface in their conceptual model, but their experimental data were used to assess column-scale mass transfer coefficients and they did not need to separately consider interfacial processes.

Efforts to quantify SVE either consider the details of microscale processes occurring at the soil grain and water film scales (Hayden and Voice, 1993; Wilson, 1990), or they parameterize those processes for representation at the meter scale that is typical of numerical solution procedures (Armstrong et al., 1994). Comparison of model predictions with long-term field measurements has been limited. Under such circumstances, it is important to consider all possible transport processes and evaluate their importance at a range of scales to see if inclusion within full-scale models is justified. Volatile NAPL spreading at the air-water interface within the vadose zone was therefore considered in this effort because this mechanism has not been quantitatively addressed in prior literature.

Depending on the interfacial tensions among the air, water and NAPL, spreading NAPLs will spread in a film on the air-water interface, and nonspreading NAPLs will become trapped in larger pores and coalesce into lenses on the air-water interface. NAPLs that spread in the subsurface will have a greater air-NAPL surface area, which will enhance mass transfer into the soil gas (Anderson, 1994; Wilson, 1990) and the SVE efficiency.

The spreading coefficient $(S)$ describes the tendency of oil to spread on the air-water interface (Adamson, 1990) and is given by

$$
S=\sigma_{\mathrm{aw}}-\left(\sigma_{\mathrm{ow}}+\sigma_{\mathrm{oa}}\right)
$$

where $\sigma$ is interfacial tension; subscripts a, w, and o refer to the air, water, and oil phases, respectively; and pairs of subscripts refer to the interface between the respective phases. If $S$ is positive, the oil spreads spontaneously; if negative, the oil beads up into lenses. For example, at $20^{\circ} \mathrm{C}$, water has an interfacial tension with air of $0.0729 \mathrm{~N} / \mathrm{m}$ (Jasper, 1972), and hexane has a reported interfacial tension of $0.0184 \mathrm{~N} / \mathrm{m}$ with air and $0.0511 \mathrm{~N} / \mathrm{m}$ with water (Demond and Lindner, 1993), yielding an $S$ of $0.0034 \mathrm{~N} / \mathrm{m}$ for pure water and hexane phases. Interfacial tensions are dependent upon oil and water partitioning between the two liquid phases and the presence of other compounds at the air-water interface, often resulting in lower spreading coefficients after mutual saturation. The value of the initial spreading coefficient may be positive, negative or zero, but the value of the equilibrium spreading coefficient may only be zero or negative (Hirasaki, 1993). Because of this, spreading of oil on water in a closed container may occur until all available surfaces are covered, or an equilibrium water-oil-air configuration is attained, at which point spreading stops. The vadose zone is not closed, however, particularly under SVE conditions, which draw the system away from equilibrium. Flow of soil gas will tend to dilute the concentration of the volatile oil in the soil gas. This will 
reduce the partitioning to the air-water interface and increase the spreading coefficient compared to the equilibrium value (Bruant and Conklin, 2001; Hauxwell and Ottewill, 1968, 1970; Pennell et al., 1992). Volatilization under non-equilibrium conditions will reduce the significance of monomolecular films that extend from NAPLs. Here, we will not address monomolecular films, as they are not expected to play a large role in mass transfer.

The spreading tendency of some oil mixtures on the air-water interface has been observed to change from negative to positive (nonspreading to spreading) over time (Schroth et al., 1995). This may be due to changes in the composition of the multicomponent oil since individual components may volatilize or dissolve. Interfacial tensions also change with the temperature and the presence of other compounds (i.e. surfactants). Interfacial tension gradients are known to cause spreading and this is called the Marangoni effect (Ahmad and Hansen, 1972).

The static equilibrium condition of a spreading lighter-than-water NAPL (LNAPL) on capillary-held water on a rough water-wet surface can be described by pendular layers of oil filling indentations created by capillary-held water. We use the term "corner" to allow for a better description of the roughness of natural surfaces and the geometry of particle contacts. In corners, layers of capillary-held liquids are thicker than on flat or convex surfaces. These oil layers in corners could be present to a height $\left(h_{\text {corner }}\right)$ on the order of

$$
h_{\text {corner }}=\frac{h_{0}}{\Gamma-1}
$$

above the location where the oil pressure is equal to atmospheric pressure (Blunt et al., 1995; Kantzas et al., 1988). In Eq. (2)

$$
\Gamma=\frac{\rho_{\mathrm{w}}-\rho_{\mathrm{a}}}{\rho_{\mathrm{w}}-\rho_{\mathrm{o}}} \frac{\sigma_{\mathrm{ow}}}{\sigma_{\mathrm{oa}}}
$$

and $h_{\mathrm{O}}$ is the thickness of the oil pool, defined as the vertical distance between the location where the air and oil pressures are equal and the location where the oil and water pressures are equal. Eq. (2) is valid for spreading LNAPLs and only when the value of $\Gamma$ is greater than 1 .

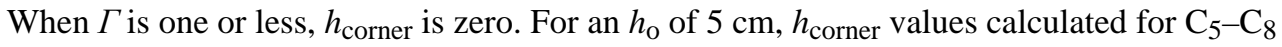
alkanes using published densities and interfacial tensions (Demond and Lindner, 1993) are approximately $1 \mathrm{~cm}$. With spreading oils, pendular oil overlying the water film is connected by oil films, and above $h_{\text {corner, }}$, oil may still exist in films. Eqs. (2) and (3) provide important insights into spreading oil behavior, but they consider only the case of thermodynamic equilibrium in a closed system, and do not consider interfacial tension imbalances. Drainage through pendular oil elements for spreading and nonspreading nonvolatile oils has been theoretically considered and experimentally evaluated (Blunt et al., 1995; Oren et al., 1992b; Oren and Pinczewski, 1995; Soll et al., 1993; Zhou and Blunt, 1997). Enhanced drainage was observed through connected oil films situated between the water and gas phases.

Film spreading velocities are orders of magnitude faster on a pool of water compared to a waterwet porous medium (Davies and Rideal, 1963). For example, the surfactant 1-tetradecanol spread at $26.5 \mathrm{~cm} / \mathrm{s}$ on a flat air-water interface but only about $3 \mathrm{~cm} /$ day in a water-wet porous medium (Tschapek and Wasowski, 1982). Spreading rate relations have been derived or observed for many free surface conditions, particularly for monolayers on viscous liquid substrates. Although we consider thicker spreading films in this study, it is important to be aware of these spreading phenomena, because there are important similarities. Ahmad and 
Hansen (1972) derived a simple relation for monolayer spreading velocity for steady-state film flow on a horizontal liquid surface:

$$
x^{2}=\left(2 d \pi_{o} / \mu\right) t
$$

In this relation, $x$ is the distance to the observed film front, $d$ is the thickness of the substrate liquid, $\pi_{\mathrm{o}}$ is the spreading pressure (defined as the interfacial tension without the monolayer minus the interfacial tension with the monolayer), $\mu$ is the substrate film viscosity and $t$ is time. In their experiments using oleic acid spreading over glycerol, good agreement between their relation and observations was obtained for thick substrates. They also observed a very noticeable height depression of the substrate film in the neighborhood of the source, leading to a "dry" area surrounding the source for thin substrate layers and a sharp variation in substrate layer thickness at the boundary.

Davies and Rideal (1963) present a three-region curve describing the spreading velocity over a thick liquid substrate of limited surface area. The initial spreading is dependent on the thickness of the applied oil (region 1), after which steady spreading occurs (region 2) until depletion, desorption or evaporation become important. The final region (region 3 ) is slower because other processes such as evaporation are important.

Spreading behavior in other systems provides insights into NAPL spreading on a water film. Fraaije and Cazabat (1989) noted that polydimethylsiloxanes (PDMS) spreading over glycerol was considerably faster on thicker glycerol films. They proposed a spreading relation similar to the one presented by Ahmad and Hansen (1972) to describe spreading of the monomolecular precursor film. They concluded that only the spreading of the precursor film (a very thin film preceding thicker spreading films) was dependent on the spreading pressure, and the spreading of the oil drops themselves was related to gravitational and capillary forces because the precursor film "burns up" the spreading power. They also noticed that when PDMS was placed on $0.2 \mathrm{~cm}$ thick glycerol layers, the glycerol "retracted vehemently" leaving a "completely dry halo", which was later invaded again by the glycerol and spreading continued. When the PDMS was placed on glycerol layers $0.1 \mathrm{~cm}$ and thinner, the glycerol no longer reinvaded the dry region and the PDMS continued to spread slowly on the solid surface previously covered by glycerol.

Gaver and Grotberg (1992) studied fluid flow within a glycerol substrate layer when oleic acid spread over the surface. They noted that when the ratio of the restoring gravitational force to the surface tension gradient was less than 0.5 , thinning of the substrate film would occur and rupture was possible. In an experiment with a substrate thickness of $0.4 \mathrm{~mm}$, the spreading oleic acid caused a dry region to form around the oleic acid droplet. Beyond that, some oleic acid film spreading had occurred but that was halted when the dry region formed. We interpret this to mean the dry region inhibits further spreading, because spreading over a solid is relatively slow. Jensen and Grotberg (1992) evaluated film rupture for spreading films. They concluded that the spreading reduced the substrate liquid film thickness to a limiting thickness where van der Waals forces cause the rupture. Hirasaki (1991) explains that two minimum stable substrate film thicknesses may be possible depending on the composition of the disjoining pressure components and initial conditions. A thick film will thin first to the thicker stable configuration with further thinning to the thinner stable thickness requiring additional energy. A film starting from zero thickness will thicken to the lower stable thickness and will not become thicker. We interpret this to mean that for the initially thicker layers $(0.2 \mathrm{~cm})$ used by Fraijje and Cazabat, the substrate layer thinned to the thicker stable configuration, and then thickened again over time. For the thinner initial substrate layers $(0.1 \mathrm{~cm})$, the thinner stable 
film thickness may have occurred during spreading providing a configuration that could not return to the thicker substrate film condition.

McBride et al. (1989) measured the pressure (suction) necessary to keep an oil film from spreading on an air-water interface in a beaker (thick substrate). Two oils were used, Soltrol-220 and diesel. Although the Soltrol-220 had a negative spreading coefficient, a pressure of $45 \mathrm{~cm}$ of water was required to stop the Soltrol from spreading. For diesel, which had a positive spreading coefficient, a pressure of about $340 \mathrm{~cm}$ of water was required to prevent the diesel from spreading. Upon spreading, characteristic light interference patterns were observed, indicating the formation of films thicker than monolayers.

In laboratory three-phase (water-oil-air) systems in porous media and micromodels used to investigate contaminant transport and enhanced oil recovery, flow in films has explained rapid migration of spreading oils (Blunt et al., 1995; Keller et al., 1997; McBride et al., 1992; Oren et al., 1992a; Soll et al., 1993; Wilson et al., 1990). Within the vadose zone, NAPL spreading at the air-water interface is expected but the effects of oil spreading on SVE have not been previously quantified. This paper presents an experimental investigation into NAPL spreading in water-wet porous media for a volatile oil over a range of laboratory scales.

\section{Conceptual model for oil spreading}

We modeled oil spreading from a NAPL pool present on a static water table. Above the pool, NAPL is drawn upwards on the air-water interface by an imbalance in interfacial tensions in what we call a film zone. Above the film zone is the vapor zone where gas-phase advective and diffusive mass transport dominate. The film and vapor zones are similar to the conceptual model for water evaporation through soil columns used by Gardner (1958).

When air is drawn through a region containing a volatile NAPL, enhanced volatilization is expected over passive diffusion. Gas-phase concentrations in the extracted air down-stream of a pool of NAPL on the water table are compared for a spreading and a nonspreading oil as illustrated in Fig. 1a and b, respectively. Although gas flow through the subsurface typically occurs in three dimensions, we consider here only steady-state one-dimensional horizontal soil gas transport and vertical dispersion through a uniform porous medium described by:

$$
U_{x} \frac{\partial C}{\partial x}=D_{z} \frac{\partial^{2} C}{\partial z^{2}}
$$

where $U_{\mathrm{x}}$ is the horizontal air approach velocity, $C$ is the gas-phase concentration of the oil and $D_{\mathrm{z}}$ is the transverse dispersion coefficient, defined as:

$$
D_{z}=D_{\mathrm{AB}, \mathrm{pm}}+\alpha_{\mathrm{t}} \frac{U_{x}}{n_{\mathrm{a}}}
$$

where $\alpha_{\mathrm{t}}$ is the transverse dispersivity, $D_{\mathrm{AB}, \mathrm{pm}}$ is the tortuosity-corrected diffusion coefficient, and $n_{\mathrm{a}}$ is the air-filled porosity. The diffusion coefficient can be corrected for moisture content using the Millington-Quirk relationship (Jury et al., 1991):

$$
D_{\mathrm{AB}, \mathrm{pm}}=\frac{n_{\mathrm{a}}^{10 / 3}}{n^{2}} D_{\mathrm{AB}}
$$

where $n$ is the porosity. 
The boundary conditions for a nonspreading oil are a zero inlet concentration at $\mathrm{x}=0$, the gasphase oil concentration immediately above the oil pool is saturated $\left(\mathrm{C}_{\mathrm{sat}}\right)$ and there is no source or sink of oil as $z$ approaches $\infty$. Under these conditions, the average concentration of soilvapor extracted for a horizontal extent of NAPL contamination, $L$, for nonspreading oil, $C_{\mathrm{avg}, \mathrm{ns}}(L)$, is (Hunt et al., 1988,1989):

$$
C_{\text {avg,ns }}(L)=C_{\text {sat }}\left\{\operatorname{erfc}(\omega)+\frac{1-\exp \left(-\omega^{2}\right)}{\omega \sqrt{\pi}}\right\}
$$

where

$$
\omega=\frac{h}{2\left(D_{z} L / U_{x}\right)^{1 / 2}}
$$

and $h$ is the thickness of the unsaturated zone. The variable $\omega$ is the ratio of the vadose zone thickness to the distance a contaminant would diffuse in the contact time available.

In the case of a spreading oil with a film height of $h_{\mathrm{f}}$, the gas-phase concentration is expected to be $C_{\text {sat }}$ within the film zone. Above the film zone is a concentration distribution identical to that over a nonspreading NAPL pool. The average concentration in the extracted air is then calculated considering the concentration profile for the nonspreading case above the film zone and $C_{\text {sat }}$ within the film zone:

$C_{\mathrm{avg}, \mathrm{s}}=\frac{C_{\mathrm{sat}}}{\omega}\left(\left(\omega-\omega_{\mathrm{f}}\right) \operatorname{erfc}\left(\omega-\omega_{\mathrm{f}}\right)+\frac{1-\exp \left[-\left(\omega-\omega_{\mathrm{f}}\right)^{2}\right]}{\sqrt{\pi}}+\omega_{f}\right)$

where

$$
\omega_{\mathrm{f}}=\frac{h_{\mathrm{f}}}{2\left(D_{z} L / U_{x}\right)^{1 / 2}}
$$

The ratio of the spreading to the nonspreading concentration is an enhancement factor, $\eta$, which from Eqs. (8) and (10) gives

$$
\eta=\frac{\omega_{\mathrm{f}}+\left(\omega-\omega_{\mathrm{f}}\right) \operatorname{erfc}\left(\omega-\omega_{\mathrm{f}}\right)+\frac{1}{\sqrt{\pi}}\left\{1-\exp \left[-\left(\omega-\omega_{\mathrm{f}}\right)^{2}\right]\right\}}{\omega \operatorname{erfc}(\omega)+\frac{1}{\sqrt{\pi}}\left\{1-\exp \left[-\omega^{2}\right]\right\}}
$$

Eq. (12) is plotted in Fig. 2. To evaluate the enhancement factor, estimates for the height of film zones are required. We convert the pressures measured by McBride et al. (1989) needed to keep diesel and Soltrol-220 from spreading. Considering fluid densities (0.84 and 0.8, respectively) these pressures correspond to 404 and $56 \mathrm{~cm}$ of oil, respectively, and films zones with these heights could result. We will assume that the air approach velocity $\left(U_{\mathrm{x}}\right)$ is $10 \mathrm{~m} / \mathrm{d}$, the horizontal extent of NAPL contamination $(L)$ is $15 \mathrm{~m}$, the vadose zone thickness is $5 \mathrm{~m}$, the porosity is $35 \%$ and the air-filled porosity is $30 \%$. We use hexane's molecular diffusion coefficient $\left[7.32 \times 10^{-6} \mathrm{~m}^{2} / \mathrm{s}\right.$ (Yaws, 1995)] as representative. 
For our example calculation, we ignore the hydrodynamic component of transverse dispersion. Hydrodynamic dispersion is expected to contribute to longitudinal dispersion when the molecular Peclet number $\left(U d_{\mathrm{g}} / D_{\mathrm{AB}}\right.$ where $d_{\mathrm{g}}$ is the grain diameter) is greater than about 1 and the dominance of hydrodynamic dispersion in the transverse direction requires a molecular Peclet number an order of magnitude larger according to studies in water-saturated systems (Perkins and Johnston, 1963). For dispersion in the gas phase, a similar dependence on molecular Peclet number is expected based on experimental results for longitudinal dispersion observed by Popovicová and Brusseau (1997). Using a 1-mm grain diameter and the diffusivity for hexane, gas velocities of over $6000 \mathrm{~m} /$ day are required for the transverse dispersion to be significant.

For the parameters listed, $\omega_{\mathrm{f}}$ is 1.34 for a film height of $1 \mathrm{~m}$ (an arbitrary height between 56 and $404 \mathrm{~cm}$ suggested by the work of McBride et al., 1989), and $\omega$ is 6.7. In Fig. 2, we see that the increase in efficiency of SVE is improved a factor of 3.4 by the presence of the $1 \mathrm{~m}$ film zone (Point A in Fig. 2). For a film height of $0.5 \mathrm{~m}$, the enhancement is 2.18 (Point B in Fig. 2) and, for a film height of $4 \mathrm{~m}$, the enhancement is 10.44 .

For a film height of $1 \mathrm{~m}$ and a vadose zone just thicker than the film height ( $\omega$ just greater than 1.34 in this case), there is some enhancement $(\eta=2.44)$. As the vadose zone thickness increases, the enhancement also increases, reaching a maximum value of about 3.4. This is somewhat counter-intuitive, as one might expect the greatest increase in enhancement to occur when the film height is equal to the vadose zone height $\left(\omega_{\mathrm{f}}=\omega\right)$ and $C_{\mathrm{s}}$ is equal to $C_{\mathrm{sat}}$. This does not occur because dispersion increases $C_{\mathrm{ns}}$ relative to $C_{\text {sat }}$, thus the ratio of $C_{\mathrm{s}}$ to $C_{\mathrm{ns}}$ is not at its maximum there.

The models in Eqs. (8) and (10) for spreading and nonspreading conditions both assumed diffusion into a semi-infinite porous medium. This assumption is reasonable for thick vadose zones but only approximate as the vadose zone thickness approaches the characteristic distance for diffusion in the advection time available, which is $\left(2 D_{\mathrm{z}} L / U_{\mathrm{x}}\right)^{1 / 2}=0.53 \mathrm{~m}$ under the adopted geometry and flow conditions.

This analysis and extensions from the literature predict that film spreading will enhance SVE efficiency in cases where the water films are thick (and oil spreading is faster), where the soil vapor is moving rapidly, and in deeper vadose zones. In practical situations, thicker water films are found near the water table resulting in slower soil vapor velocities where increased water content reduces gas-phase permeability.

\section{Experimental investigation}

Several scoping-level experiments were performed to gain insights into oil spreading on flat surfaces and in porous media. The experimental investigation of film spreading in water-wet porous media was then scaled up to quantitatively evaluate oil film spreading and volatilization.

\subsection{Oil spreading verification}

Spreading oils were introduced onto horizontal, inclined, and vertical water layers. Glass petri dishes and a larger glass plate were cleaned using a commercial laboratory detergent (Sparkleen ${ }^{\circledR}$ ) and rinsed thoroughly (in cold tap water, triple rinsed in hot tap water, triple rinsed in cold tap water, triple rinsed in distilled water and triple rinsed in distilled-deionized water) until a thick (submillimeter) water layer remained on the glass following inclination. Several volatile oils [hexane, $\mathrm{S}=0.003 \mathrm{~N} / \mathrm{m}$ (MCB Reagents OmniSolv), heptane, $S=0.0027$ $\mathrm{N} / \mathrm{m}$ (Fisher HPLC Grade)] and nonvolatile oils [Liquid Wrench, $S=0.0371 \mathrm{~N} / \mathrm{m}$ (Radiator Specialty, Charlotte, NC), S.A.E. 20 motor oil, $S=0.0034$ N/m (Boyle-Midway, New York, $\mathrm{NY}$ )] were selected based on their initial spreading coefficient calculated from published 
interfacial tensions (e.g. Demond and Lindner, 1993; Hirasaki, 1993; Mercer and Cohen, 1990; U.S. Department of Transportation and U.S. Coast Guard, 1984). The oils were placed using a dropper into the water pool created in the lip of the petri dish by the draining water, and the resulting spreading behavior was visually observed. The nonvolatile oils spread rapidly up the inclined and vertical water layers to the top of the water layer on the glass $(10$ to $15 \mathrm{~cm}$ ), thinning the water layer above the water pool significantly (in a band about $6 \mathrm{~mm}$ high for the Liquid Wrench, and about $25 \mathrm{~mm}$ for the motor oil), with the water layer rupturing during motor oil spreading. The oil film was visually observed as a sheen over the water layer indicating an oil film thickness much thicker than a monolayer. The area where water layer rupture occurred did not become water-wet following immersion of the glass in water. Volatile spreading oils also spread rapidly and caused water-layer rupture, however, the ruptured water layer became water wet following oil volatilization. Again, the oil layers were thick enough to be detected visually. The volatile oils did not spread to the vertical extent of the water film because evaporation occurred before reaching that point.

\subsection{Effect of moisture content on spreading in a porous medium}

Film flow speeds increase with the thickness of the liquid substrate (Ahmad and Hansen, 1972; Fraaije and Cazabat, 1989). To investigate this effect in a porous medium, moisture content was used as a surrogate for water film thickness. A volume of $0.3 \mathrm{ml}$ of red dyed heptane was introduced into $18 \mathrm{~mm}$ diameter $\times 150 \mathrm{~mm}$ test tubes containing $\sim 375$ micron glass beads (Cataphote, Jackson Mississippi) with moisture contents ranging from $0.0002 \mathrm{~g}_{\text {water }} /$ $\mathrm{g}_{\text {dry glass }}$ (air dry) to 0.0816 determined gravimetrically. The tubes were sealed with cork stoppers covered with Teflon and maintained horizontally. A needle was used to inject the oil through the cork resulting in a small hole through the stopper. In the driest sample, a pink region formed around the liquid pool after about $30 \mathrm{~min}$. After about $20 \mathrm{~h}$, a band of dull red dye, indicating dry dye without heptane, was observed towards the cork end of the tube about $0.5 \mathrm{~cm}$ from the liquid pool location. This band became more intensely colored over time indicating that liquid heptane was transporting dye from the liquid pool to the band where heptane volatilization occurred. At moisture contents from 0.0049 to 0.0197 , very little heptane movement was observed over the course of a week. At moisture contents from 0.0274 to 0.0816 , heptane movement was immediately observable with velocities on the order of centimeters per hour and the velocities increased with increasing moisture content. Thus, two thresholds were observed; slow heptane spreading below a moisture content of 0.0049 and rapid spreading above a moisture content of 0.0274 . At the lowest moisture contents, heptane was probably spreading on the glass surface and insufficient water was present to stop it. At intermediate water contents, heptane may have initially spread on the water film and caused water film rupture which hindered further spreading. In this case, the heptane and water films would both be trying to spread against each other on the glass surface resulting in nondetectable movement. At the higher moisture contents, heptane was probably spreading on continuous water films. Pendular rings in a bed of spheres join at water contents of approximately 0.035-0.07 corresponding to our wettest conditions (Haines, 1926). Film spreading on these joined pendular rings at higher water contents may be responsible for the more rapid spreading.

\subsection{Capillary tubes}

To examine film spreading in a simple geometry containing corners, film spreading and vapor diffusion were examined in three square capillary tubes. The cleaned tubes were $23 \mathrm{~cm}$ long with a square cross section having $1 \mathrm{~mm}$ sides and $0.05 \mathrm{~mm}$ corner radii. Contrary to a porous medium, where the contact points between grains allow very small radii of curvature of the air-water interface, capillary-held water in the tubes would be present in the $0.05 \mathrm{~mm}$ corners only to a height of about $14 \mathrm{~cm}$. This water layer would be much thicker than on the flat surfaces inside the tubes and would be expected to provide greater lubrication for a spreading oil film. Tube 1 was first filled with water and partially drained. Hexane was injected at the air-water 
interface through a long needle resulting in $3 \mathrm{~cm}$ of water on the bottom and $3.1 \mathrm{~cm}$ of hexane. In Tube 2, hexane was added at the top of a water-filled tube as the water was allowed to partially drain from the bottom leaving $1.5 \mathrm{~cm}$ of water and $2.5 \mathrm{~cm}$ of hexane at the bottom. In Tube 3, about $6 \mathrm{~cm}$ of hexane was placed into the bottom of a dry tube through a long needle. Clean air was swept over the tops of the tubes. For Tubes 1 and 2, the air was humidified by bubbling through a water column maintained several degrees warmer than ambient, and for Tube 3 the air was dry. Hexane levels were measured over several hundred hours with minimal loss of water.

Hexane loss in the tubes was expected through a combination of film flow and vapor diffusion. A balance between liquid loss and vapor diffusion is modeled as

$$
\rho_{\mathrm{H}} \frac{\mathrm{d} h_{\mathrm{obs}}}{\mathrm{d} t}=\frac{M_{\mathrm{H}} D_{\mathrm{Ha}} C_{\mathrm{t}}}{h_{\mathrm{v}}} \ln \left(\frac{1}{1-y_{\mathrm{sat}}}\right)
$$

which equates liquid and gaseous mass fluxes, where $\sigma_{\mathrm{H}}$ is the liquid density of hexane, $h_{\mathrm{obs}}$ is the height of the air column over the hexane liquid to the tube top, $M_{\mathrm{H}}$ is the molecular weight of hexane, $D_{\mathrm{Ha}}$ is the molecular diffusivity of hexane in air and $h_{\mathrm{v}}$ is the height of the vapor diffusion zone. Eq. (13) is used to estimate the vapor diffusion zone height $\left(h_{\mathrm{v}}\right)$ using the measured $\mathrm{d} h_{\mathrm{obs}} / \mathrm{d} t$ and then the film height is determined by difference $\left(h_{\mathrm{obs}}-\mathrm{h}_{\mathrm{V}}\right)$. Fig. 3 plots $h_{\mathrm{obs}}$ and the calculated film heights during volatilization. For Tubes 1 and 2 , the liquid flux of hexane was constant over time, indicating a steady increase in calculated film height. These data suggest that the boundary of hexane spreading was pinned at a fixed location, with the film formed by drainage in Tube 2 being higher and the film formed by spreading in Tube 1 being lower. For Tube 3, a slight curvature is seen in the data indicating that as the hexane volatilized, $h_{\mathrm{v}}$ increased as expected. In Tube 1 , the hexane film had to spread on an initially hexane-free air-water interface, likely resulting in rapid spreading. This rapid spreading may have induced water film rupture and the pinning of the contact line. In Tube 2, hexane likely spread as the drainage occurred resulting in slower spreading. As continued slower film spreading occurred, the thinner water film present at a higher elevation may have been further thinned by the spreading inducing water film rupture and pinning of the contact line at an elevation exceeding that in Tube 1 .

\subsection{Meter-scale column experiments}

To evaluate film spreading on a larger scale, experiments were conducted in meter-tall columns shown in Fig. 4. The glass tubes were $3.4 \mathrm{~cm}$ in diameter and packed with approximately 100 $\mathrm{cm}$ of water-wet glass beads or sand. The columns were initially wetted by filling with water from the bottom and then allowing the water to drain over several days, leaving a watersaturated zone at the bottom. Column temperatures were controlled to within a few degrees Celsius by flowing temperature-controlled water through tubes surrounding the columns and insulating the resulting system. Hexane was introduced above the water-saturated zone either at a constant head with a Mariotte bottle or at a constant loading rate with a syringe pump. The syringe pump was used to supply the spreading oil very slowly in an attempt to reduce the possibility of water film rupture. In both methods of hexane introduction, a liquid hexane zone was eventually observed above the water-saturated zone. Humidified nitrogen was used as the sweep gas and was introduced at locations A, B or C indicated in Fig. 4.

Table 1 provides a summary of conditions and results for the 6 experiments conducted in the meter-tall columns. In Experiments 1, 2 and 3, sweep gas was introduced at different times at three separate locations (A, B and C) designated in Fig. 4. Location A had the sweep gas introduced near the bottom of the porous medium column to simulate a shallow vadose zone, location B was at an intermediate height and location $\mathrm{C}$ has the sweep gas flushed across the 
top of the porous media. In Experiments 4, 5 and 6, the sweep gas was only introduced across the top of the porous medium at location $\mathrm{C}$. Hexane flux through the columns was determined from measurements of the effluent concentration and effluent flow rate. Additionally, after reaching steady-state conditions when sweep air was introduced at location $C$, soil gas hexane measurements were taken from locations A and B to determine the profile of hexane within the column. Steady-state conditions were defined as (1) a period exceeding the predicted time to reach $99 \%$ of steady state, (2) no apparent trend in the data and (3) the standard deviation of the collected data was generally less than $5 \%$ of the average. Gas samples were withdrawn first from location $\mathrm{C}$, then $\mathrm{B}$ and then from $\mathrm{A}$ to minimize disruption of the concentration profiles. Prior to sampling, the sampling tube was flushed out by wasting more than three times the sampling tube volume. Hexane was quantified by direct injection of gas into a gas chromatograph. At the end of the experiment, moisture contents were determined gravimetrically by sectioning and sampling. Some contact with the atmosphere occurred during sampling and it was assumed that any volatile NAPL present was evaporated thus not impacting the gravimetric analysis. For Experiments 4 and 5, liquid hexane was introduced at a steady rate by a syringe pump at a flux approximately double the flux predicted for a nonspreading liquid having identical properties to hexane. This rate would have been sufficient for film spreading to occur. Additional details on materials, assembly, sampling, cleaning and calibration are found in Kneafsey (1996).

3.4.1. Film height determination-The top of the liquid hexane zone was visually observable and thus the distance from that location to the location where sweep air was introduced represents the sum of the film zone and the vapor zone. The height of the vapor zone was quantified by two different methods: the Effluent Method and the Profile Method, and then the film height was determined by difference.

3.4.1.1. Effluent method: The mass flux equation

$$
F_{\mathrm{H}}=-C_{\mathrm{tot}} D_{\mathrm{Ha}} \frac{\mathrm{d} y_{\mathrm{H}}}{\mathrm{d} x}+y_{\mathrm{H}}\left(F_{\mathrm{H}}+F_{\mathrm{a}}\right)
$$

is solved for the hexane profile. In Eq. (14), $F_{\mathrm{H}}$ is the molar flux of hexane, $F_{\mathrm{a}}$ is the flux of air, $C_{\text {tot }}$ is the total gas concentration $\left(C_{\text {tot }}=P_{\text {tot }} / R T\right)$ where $P_{\text {tot }}$ is the total pressure, $R$ is the gas constant and $T$ is absolute temperature. $D_{\mathrm{Ha}}$ is the diffusion coefficient of hexane in the binary hexane-air system and $y_{\mathrm{H}}$ is the mole fraction of hexane in the gas phase.

We assume that the vertical air flux is negligible $\left(F_{\mathrm{a}}=0\right)$ within the packed portion of the column below the introduced sweep gas, thus the height of the vapor diffusion zone is

$$
h_{\mathrm{V}}=\frac{D_{\mathrm{Ha}, \mathrm{pm}} C_{\mathrm{tot}}}{F_{\mathrm{H}}} \ln \left(\frac{1-y_{\mathrm{H}, \mathrm{e}}}{1-y_{\mathrm{H}, \mathrm{sat}}}\right)
$$

where $y_{\mathrm{H}, \mathrm{e}}$ is the measured effluent hexane mole fraction, $y_{\mathrm{H} \text {,sat }}$ is the saturated hexane mole fraction and $F_{\mathrm{H}}$ is the hexane flux determined from the sweep gas flow rate and effluent hexane concentration.

The diffusion coefficient for each column was determined by measuring either octane (Experiments 1, 2 and 3) or hexane (Experiments 4, 5 and 6) diffusive fluxes without water present. The method of Fuller (Reid et al., 1987) was used to estimate hexane diffusivities from the octane data. The hexane diffusion coefficient was corrected for moisture content using the Millington-Quirk relationship (Eq. (7)). For variable water contents, each moisture content 
measurement was applied over the region halfway up and halfway down to the next measurement and the overall correction was made applying conductances in series.

3.4.1.2. Profile method: Nearly linear mole fraction profiles would be expected for hexane in soils with constant moisture content (Baehr and Bruell, 1990). In the Profile Method, the gasphase hexane mole fraction was measured at locations A and B within the column and in the column effluent. The mole fractions were plotted versus location and a line was fitted to the data using linear regression. The line was extrapolated to the saturated mole fraction, which is assumed to be at the top of the film zone. The film zone height was then determined by difference.

3.4.2. Results - The large-scale columns reached steady state after 50-600 h. One example of the effluent gas measurements is given in Fig. 5 for Experiment 5 . Over the first $150 \mathrm{~h}$, there was an increasing hexane flux as the system reached the steady-state flux of $1.59 \times 10^{-9} \mathrm{~mol} /$ $\mathrm{cm}^{2}-$ s over the time interval of 175-300 h. The imposed hexane flux into the column bottom was $4.5 \times 10^{-9} \mathrm{~mol} / \mathrm{cm}^{2}-\mathrm{s}$ resulting in pooling of liquid hexane in the column. This liquid accumulation velocity is only about $4 \times 10^{-3} \mathrm{~cm} / \mathrm{h}$, thus a steady-state analysis is justified. In Experiments 1, 2 and 3, the time to reach steady state was approximately $100 \mathrm{~h}$ when gas was swept across the top of the column at location $\mathrm{C}$ and less than $50 \mathrm{~h}$ when air was introduced at locations A and B. For Experiment 1A, steady state was not achieved over a 200-h period.

Film heights calculated by the Effluent Method are given in Table 1 and indicate only three experiments (1C, 4 and 6) where the film height was significantly greater than zero. The standard deviations presented incorporate the error in measuring the diffusion coefficients and the error in the flux measurements. In Experiments $1 \mathrm{C}$ and 6, the heights of the liquid hexane zones were high and difficult to determine.

The measured hexane profiles are shown in Fig. 6. The replicates in Experiments 1, 2 and 3 were taken one day apart, and the profiles for Experiments 4, 5 and 6 were collected over 710 days. In Experiment 1, the water content declined with elevation due to the presence of a large capillary fringe in the small glass bead column. In Experiment 6, the water content was not uniform with depth, however the non-uniformity was much less than in Experiment 1 . The hexane profiles shown in Fig. 6c-f are close to linear and have good reproducibility. Film heights determined by the Profile Method are indicated in the last column of Table 1 . This method only gave a nonzero film height in Experiment 5 where the film height was estimated to be $17 \pm 3 \mathrm{~cm}$.

The two methods used to quantify film heights in the large-scale column experiments were in disagreement with each other. Despite disagreements between the measurement methods, neither demonstrates that NAPL spreading at the air-water will impact SVE. The Effluent Method shows the presence of small film heights in most of the experiments, although the errors are large due to uncertainties in diffusion coefficient estimation. The Profile Method requires accurate length measurements, an accurate determination of the top of the oil zone, and uniform water saturation. These measurements are much easier to make than the diffusion coefficient. The Profile Method requires a uniform saturation, which was present in several of the columns. In spite of these difficulties, there does not appear to be significant hexane film flow in water-wetted porous media.

\section{Discussion and conclusions}

The possible importance of NAPL film spreading during SVE was investigated through an analysis of interfacial processes, models for microscale film spreading and for macroscale soil gas transport. Experimental measurements at small and intermediate scales were undertaken 
to support the analysis. NAPL spreading and film flow have not been adequately addressed, particularly regarding remediating volatile NAPLs in the vadose zone using SVE. Oil spreading at the air-water interface has received considerable attention by researchers, and spreading is expected under non-equilibrium conditions for thick water films. These conditions are likely for volatile oils floating on a capillary fringe. Oil films on the order of a meter in height could significantly alter the efficiency of SVE for the removal of a NAPL pool floating on the water table. An analytical model for horizontal advection and vertical diffusion predicted that spreading oils could be removed faster than nonspreading oils.

Small and intermediate-scale experiments within idealized porous media were performed to demonstrate volatile oil spreading on water films. Thick oil films were observed in experiments on horizontal, inclined and vertical water films. Water film entrainment and rupture was also observed in these experiments with hexane and heptane. In a porous medium at variable moisture contents, dyed heptane was visually observed to spread under low and high water content conditions, but not at intermediate contents. These results suggest water films at intermediate water contents may rupture due to oil spreading, which limits oil spreading. NAPL loss from capillary tubes also demonstrated the formation of a pinned NAPL-solid contact line that was maintained as the NAPL volatilized and the film height extended.

Within the meter-long columns containing initially water-wet media, little or no hexane spreading was observed within the uncertainties of the experimental system. The experimental data were best described by hexane volatilization from a pool floating on the water table and no significant film zone. Two methods of analysis supported this observation that hexane volatilization was not significantly enhanced by film spreading. The experimental methods were capable of determining film zones greater than $10 \mathrm{~cm}$ in height. The lack of film spreading in these meter-tall columns may have been caused by intermediate water contents providing water films that thinned and possibly ruptured by the hexane spreading. This explanation agrees with the results from small-scale porous medium tubes at variable water contents.

Another explanation for the lack of spreading films when they would otherwise be expected is that the vadose zone in our experiments acted as a closed system over the time and spatial scales of spreading. Hexane vapor could volatilize from the hexane pool and contaminate the air-water interface within the column reducing the spreading coefficient. Since the spreading coefficient indicates the magnitude of the driving force for film spreading, this reduction would diminish film spreading. If this were the case, however, spreading would not have been observed in any of the horizontal tubes containing porous media. The spreading in the horizontal test tubes may have occurred in "corners" as discussed by Blunt et al. (1995) and Zhou and Blunt (1997); however, their analysis did not identify spreading thresholds.

We expected and measured larger water content in Experiment 1 due to smaller grain diameters with more pendular structures and higher surface area. In Experiment 4, we also expected and measured higher water content due to rough grains having indentations that hold capillary water. We expected a greater enhancement to oil spreading at this water content, but this effect was not observed. In Experiment 1, the water film thickness connecting pendular structures may be no thicker than for the larger grain diameters even though the moisture content was higher due to the greater surface area. In Experiment 4, even though the rough grains hold water in indentations, protrusions from their rough surfaces would lead to locally thinner water films where water film rupture is more likely and limited flow.

While spreading was expected to be important during SVE of volatile NAPLs, the laboratory results suggest that film heights will not be significant. Experiments were limited to porous media having water drained to a residual saturation and no net movement of soil gas. Movement of soil gas could increase disequilibrium, which may enhance film spreading. Film spreading 
was observed in small-scale experiments, however, at larger scales, it did not contribute significantly to overall mass transfer. This points out the importance of examining mechanisms at various spatial scales to make sure that the essential fundamentals are retained, and nonessential mechanisms can be ignored. There may be additional information provided by micromodel studies, but quantitative scaling relationships of residual water, water film dynamics, porous medium grain sizes and NAPL spreading coefficient will need to be established. We did not investigate multicomponent NAPLs in our large-scale tests because the composition and properties of the NAPL at the evaporation front could be significantly different from the original NAPL. Multicomponent NAPLS, particularly those containing surfactants may behave differently than those used in our study and are worth investigating.

\section{Acknowledgments}

This research was supported by the NIEHS Superfund Basic Research Program, grant 3P432 ES04705-14. Discussions with Clay Radke were very much appreciated. Bruce Jacobson provided necessary and timely assistance in equipment assembly. Reviews and comments by Curt Oldenburg, Andre Unger, Martin Blunt and the anonymous reviewers were most appreciated.

\section{References}

Adamson, AW. Physical chemistry of surfaces. Wiley; New York: 1990.

Ahmad J, Hansen RS. A simple quantitative treatment of the spreading of monolayers on thin liquid films. J. Colloid Interface Sci 1972;38(3):601-604.

Anderson MA. Interfacial tension-induced transport of nonaqueous phase liquids in model aquifer systems. Water Air Soil Pollut 1994;75:51-60.

Armstrong JE, Frind EO, McClellan RD. Nonequilibrium mass transfer between the vapor, aqueous, and solid phases in unsaturated soils during vapor extraction. Water Resour. Res 1994;30(2):355-368.

Baehr AL, Bruell CJ. Application of the Stefan Maxwell equations to determine limitations of Fick's law when modeling organic vapor transport in sand columns. Water Resour. Res 1990;26(6):1155-1163.

Baehr AL, Hoag GE, Marley MC. Removing volatile contaminants from the unsaturated zone by inducing advective air-phase transport. J. Contam. Hydrol 1989;4:1-26.

Barnes DL, McWhorter DB. Design of soil vapor extraction systems under conditions of uncertainty. Ground Water 2000;38(2):209-217.

Blunt M, Zhou D, Fenwick D. Three-phase flow and gravity drainage in porous media. Transp. Porous Media 1995;20(12):77-103.

Bruant RG Jr. Conklin MH. Adsorption of trichloroethene at the vapor/water interface. Environ. Sci. Technol 2001;35:362-364. [PubMed: 11347610]

Brusseau ML. Transport of organic chemicals by gas advection in structured or heterogeneous porous media: development of a model and application to column experiments. Water Resour. Res 1991;27 (12):3189-3199.

Conrad SH, Wilson JL, Mason WR, Peplinski WJ. Visualization of residual organic liquid trapped in aquifers. Water Resour. Res 1992;28(2):467-478.

Davies, JT.; Rideal, EK. Interfacial phenomena. Academic Press; New York: 1963.

Demond AH, Lindner AS. Estimation of interfacial tension between organic liquids and water. Environ. Sci. Technol 1993;27(12):2318-2331.

Fischer U, Schulin R, Keller M, Stauffer F. Experimental and numerical investigation of soil vapor extraction. Water Resour. Res 1996;32(12):3413-3427.

Fraaije JGEM, Cazabat AM. Dynamics of spreading on a liquid substrate. J. Colloid Interface Sci 1989;133(2):452-460.

Gardner WR. Some steady-state solutions of the unsaturated moisture flow equation with applications to evaporation from a water table. Soil Sci 1958;85(4):228-232.

Gaver DP, Grotberg JB. Droplet spreading on a viscous film. J. Fluid Mech 1992;235:399-414.

Goltz MN, Oxley ME. An analytical solution to equations describing rate-limited soil vapor extraction of contaminants in the vadose zone. Water Resour. Res 1994;30(10):2691-2698. 
Haines WB. J. Agric. Sci 1926;15:529-535.

Hauxwell F, Ottewill RH. The adsorption of toluene vapor on water surfaces. J. Colloid Interface Sci 1968;28(34):514-521.

Hauxwell F, Ottewill RH. A study of the surface of water by hydrocarbon adsorption. J. Colloid Interface Sci 1970;34(4):473-479.

Hayden NJ, Voice TC. Microscopic observations of a NAPL in a three-phase soil system. J. Contam. Hydrol March;1993 12:217-226.

Hirasaki GJ. Wettability: fundamentals and surface forces. SPE Form. Eval June;1991:217-226. 1991.

Hirasaki GJ. Structural interactions in the wetting and spreading of van der Waals fluids. J. Adhes. Sci. Technol 1993;7(3):285-322.

Hunt JR, Sitar N, Udell KS. Nonaqueous phase liquid transport and cleanup: 1. Analysis of mechanisms. Water Resour. Res 1988;24(8):1247-1258.

Hunt JR, Sitar N, Udell KS. Correction to "Nonaqueous phase liquid transport and cleanup: 1. Analysis and mechanisms". Water Resour. Res 1989;25(6):1450.

Jasper JJ. The surface tension of pure liquid compounds. J. Phys. Chem. Ref. Data 1972;1(4):841-980.

Jensen OE, Grotberg JB. Insoluble surfactant spreading on a thin viscous film: shock evolution and film rupture. J. Fluid Mech 1992;240:259-288.

Johnson PC, Stanley CC, Kemblowski MW, Byers DL, Colthart JD. A practical approach to the design, operation, and monitoring of in situ soil-venting systems. Ground Water Monit. Rev 1990 Spring;: 159-178.

Jury, WA.; Gardner, WR.; Gardner, WH. Soil Physics. Wiley; New York: 1991.

Kantzas, A.; Chatzis, I.; Dullien, FAL. Mechanisms of capillary displacement of residual oil by gravityassisted inert gas injection. SPE Rocky Mountain Regional Meeting; Casper, Wyoming. 1988. p. 297-307.

Kearl PM, Korte NE, Gleason TA, Beale JS. Vapor extraction experiments with laboratory soil columns: implications for field programs. Waste Manage 1991;11:231-239.

Keller AA, Blunt MJ, Roberts PV. Micromodel observation of the role of oil layers in three-phase flow. Transp. Porous Media 1997;26:277-297.

Kneafsey, TJ. PhD dissertation. University of California, Berkeley; Berkeley, CA: 1996. The effect of vertical non-aqueous phase liquid film flow on soil vapor extraction; p. 209

Lingineni S, Dhir VK. Modeling of soil venting processes to remediate unsaturated soils. J. Environ. Eng 1992;118(1):135-152.

Massmann J, Shock S, Johannesen L. Uncertainties in cleanup times for soil vapor extraction. Water Resour. Res 2000;36(3):679-692.

McBride JF, Cary JW, Simmons CS. Direct measurement of the spreading pressure of organic liquids on water. J. Colloid Interface Sci 1989;132(2):363-372.

McBride JF, Simmons CS, Cary JW. Interfacial spreading effects on one-dimensional organic liquid imbibition in water-wetted porous media. J. Contam. Hydrol 1992;11:1-25.

Mercer JW, Cohen RM. A review of immiscible fluids in the subsurface: properties, models, characterization, and remediation. J. Contam. Hydrol 1990;6:107-163.

Oren PE, Billiotte J, Pinczewski WV. Mobilization of waterflood residual oil by gas injection for waterwet conditions. SPE Form. Eval March;1992a :70-78.

Oren PE, Billiotte J, Pinczewski WV. Mobilization of waterflood residual oil by gas injection for waterwet conditions. Soc. Pet. Eng. Form. Eval 1992b:70-78.

Oren PE, Pinczewski WV. Fluid distribution and pore-scale displacement in drainage dominated threephase flow. Transp. Porous Media 1995;20:105-133.

Pennell KD, Rhue RD, Rao PSC, Johnston CT. Vapor-phase sorption of $p$-xylene and water on soils and clay minerals. Environ. Sci. Technol 1992;26(4):756-763.

Perkins TK, Johnston OC. A review of diffusion and dispersion in porous media. Trans. Soc. Pet. Eng. Am. Inst. Min. Metall. Pet. Eng. Inc 1963;228(1):70-84.

Popovicová J, Brusseau ML. Dispersion and transport of gas-phase contaminants in dry porous media: effect of heterogeneity and gas velocity. J. Contam. Hydrol 1997;28:157-169. 
Poulsen TG, Moldrup P, Yamaguchi T, Massmann JW, Hansen JA. VOC vapor sorption in soil: soil type dependent model and implication for vapor extraction. J. Environ. Eng 1998;124(2):146-155.

Reid, RC.; Prausnitz, JM.; Poling, BE. The Properties of Gases and Liquids. McGraw-Hill; New York: 1987.

Schroth MH, Istok JD, Ahearn SJ, Selker JS. Geometry and position of light nonaqueous-phase liquid lenses in water-wetted porous media. J. Contam. Hydrol 1995;19:269-287.

Soll WE, Celia MA, Wilson JL. Micromodel studies of three-fluid porous media systems: pore-scale processes relating to capillary pressure-saturation relationships. Water Resour. Res 1993;29(9): 2963-2974.

Tschapek M, Wasowski C. Spreading of a surfactant in an unsaturated disperse material. Colloids Surf 1982;5:65-73.

U.S. Department of Transportation. U.S. Coast Guard. CHRIS hazardous chemical data. Commandant Instruction M16465.15A. 1984.

Wilkins MD, Abriola LM, Pennell KD. An experimental investigation of rate-limited volatilization in unsaturated porous media: steady state mass transfer. Water Resour. Res 1995;31(9):2159-2172.

Wilson, JL. Pore scale behavior of spreading and non-spreading organic liquids in the vadose zone. In: Weyer, KU., editor. International Conference on Subsurface Contamination by Immiscible Fluids; Calgary, Canada. A.A. Balkema; 1990. p. 107-114.

Wilson, JL.; Conrad, SH.; Mason, WR.; Peplinski, W.; Hagan, E. Laboratory Investigation of Residual Liquid Organics from Spills, Leaks, and the Disposal of Hazardous Wastes in Groundwater. USEPA; Ada, OK: 1990. p. 267

Yaws, CL. Library of physico-chemical property data. Gulf Publishing; Houston: 1995. Handbook of transport property data, viscosity, thermal conductivity, and diffusion coefficients of liquids and gases; p. 203

Zhou D, Blunt M. Effect of spreading coefficient on the distribution of light non-aqueous phase liquid in the subsurface. J. Contam. Hydrol 1997;25:1-19. 
a.

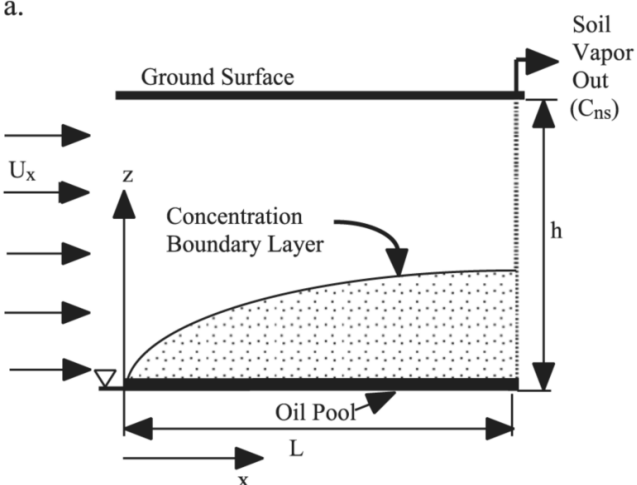

b.

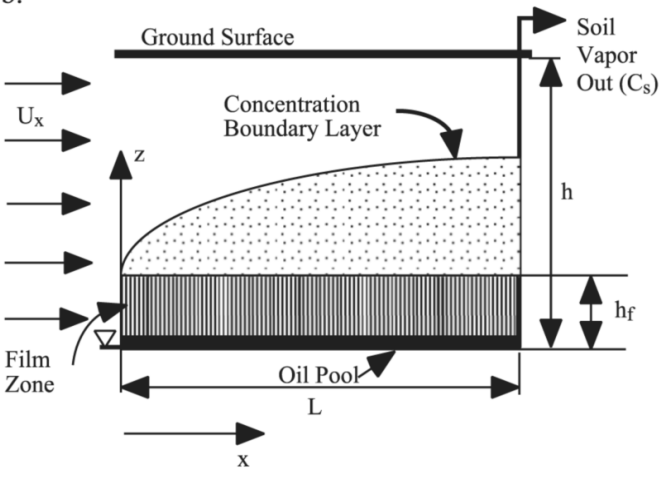

Fig. 1.

Conceptual model of development of a concentration boundary layer during soil vapor extraction for (a) nonspreading and (b) spreading cases. 


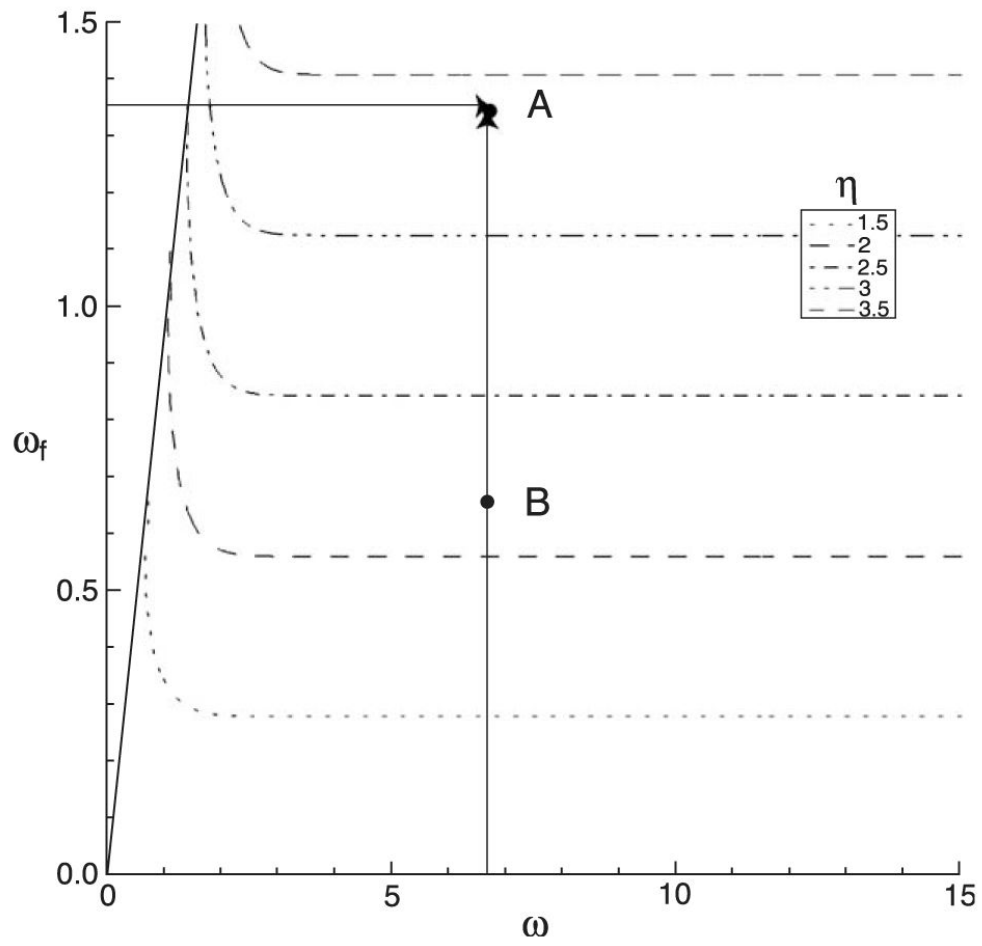

Fig. 2.

Contour plot of predicted enhancement factor for hexane recovery comparing spreading and nonspreading conditions. 


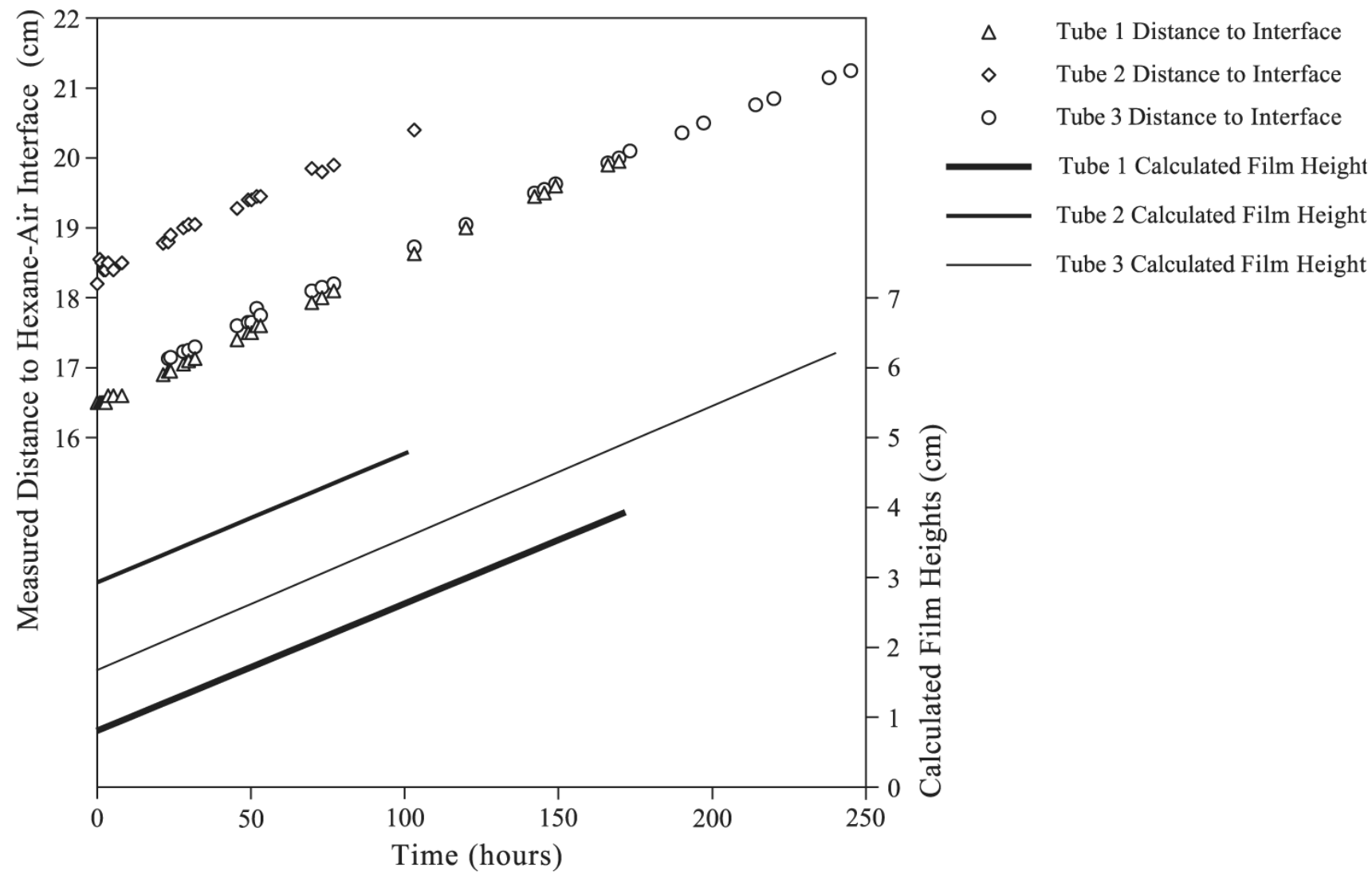

Fig. 3.

Measured distances to the hexane-air interface and calculated film heights in three capillary tube experiments. 


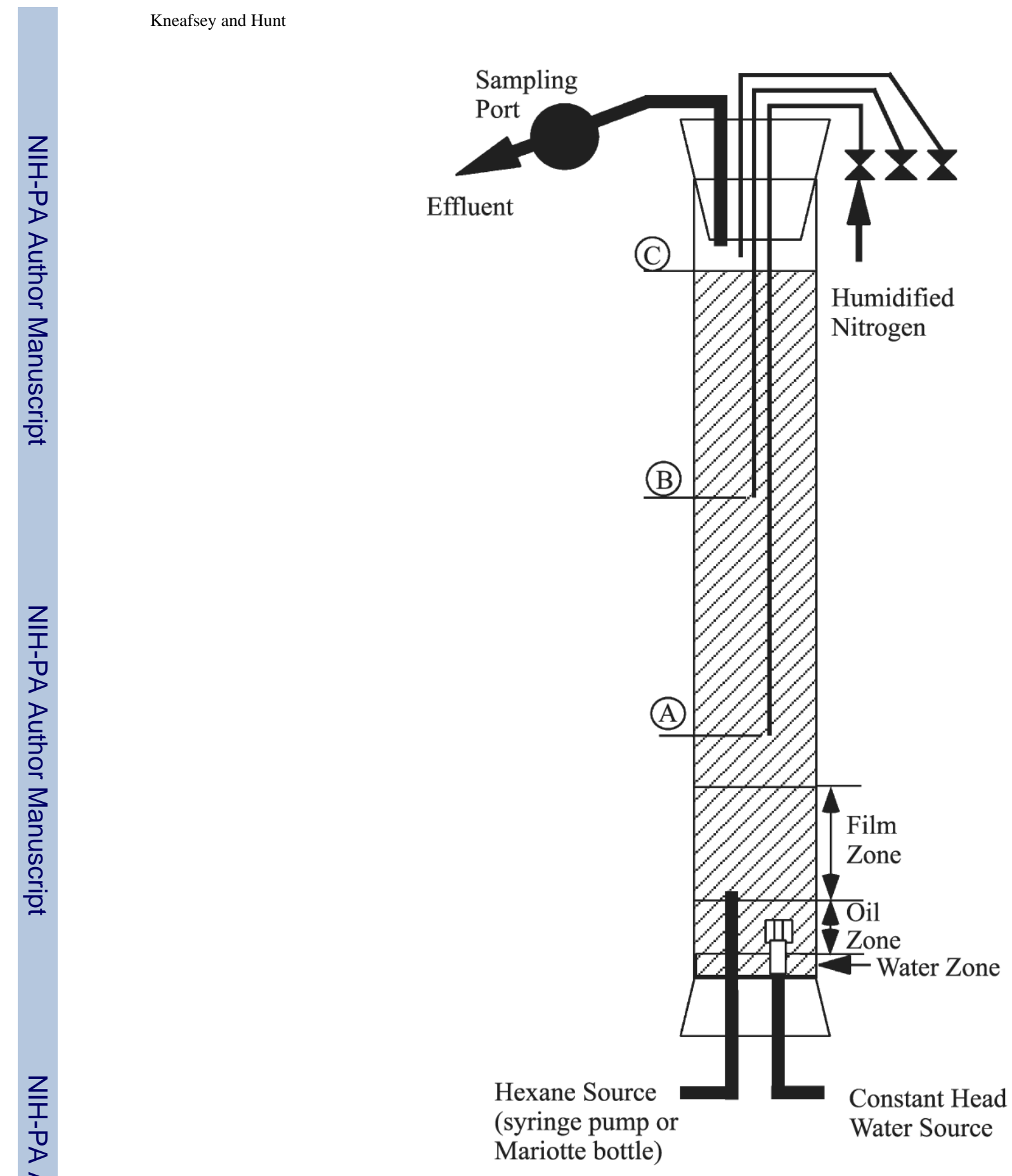

Fig. 4.

Large-scale column experiment setup. 


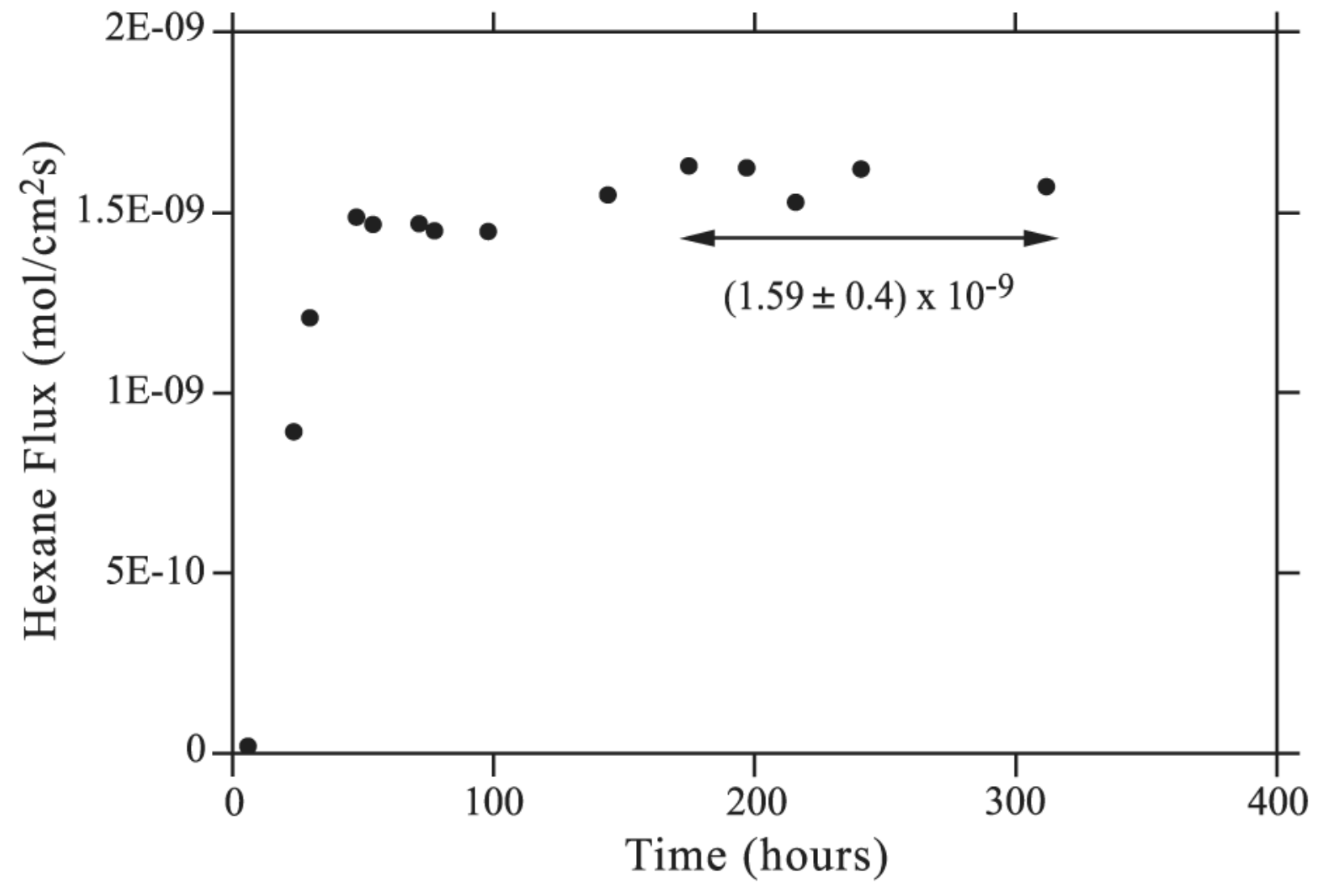

Fig. 5.

Hexane flux in Experiment 5. 
(a)

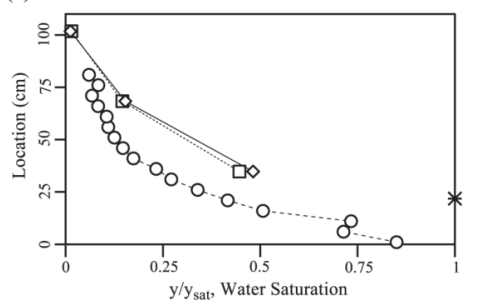

(b)

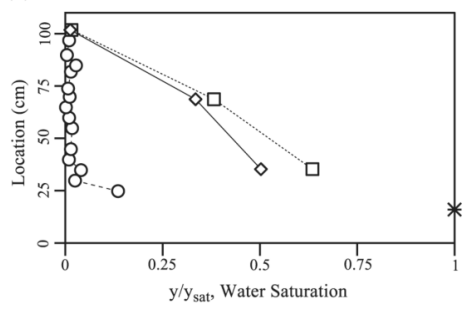

(c)

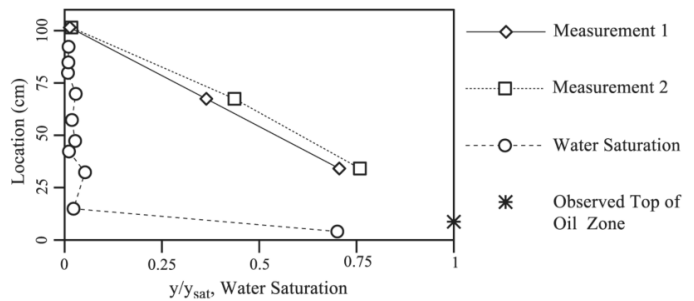

(d)

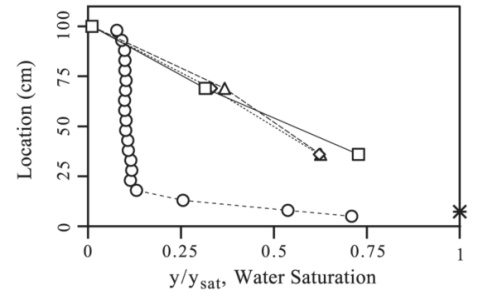

(e)

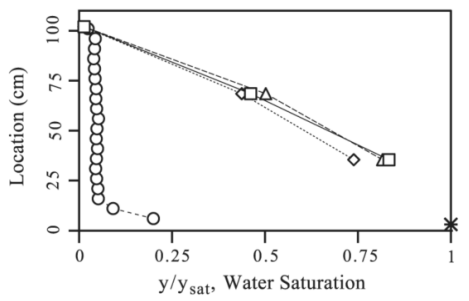

(f)

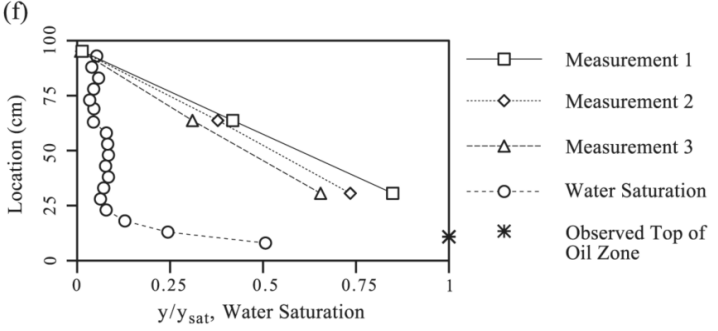

Fig. 6. 
Steady-state hexane relative mole fraction and water content profiles for sweep gas introduced at location C: (a) Experiment 1, (b) Experiment 2, (c) Experiment 3, (d) Experiment 4, (e) Experiment 5 and (f) Experiment 6. 
